\title{
Ai intelligent industrial design of Titan space probe (landing capsule and Saturn vehicle)
}

\author{
YunBo Ji \\ Luoyang Teachers College, L uoyang, Henan, 471934, China
}

\begin{abstract}
Exploring the solar system is of great significance for the realization of the community of human destiny. This article reviews the history of Saturn exploration and explores new directions in design aesthetics. The author designed a new type of Saturn's sixth satellite (Titan) with three-dimensional software 3 dsmax, including a landing capsule, a cruise vehicle (Saturn vehicle), involving the design of quantum communication, automatic driving, neural chain Ai chips, mechanical arms, etc. "Lack of beauty and tediousness", pursue "characteristics, cultural innovation". The new detector, with exquisite shapes and beautiful lines, combines the eastern cultural symbols of "Zhouyi and Dunhuang Feitian", and uses memory metal and three-dimensional printing to manufacture, expanding the new Higgs boson, neutrino, and non-carbon-based life Explore the field, named "Hetu Jishu", which closely combines mechanical aesthetics and the Silk Road.
\end{abstract}

\section{Development history of industrial design of the Saturn (satellite) probe}

\subsection{Exploring Saturn, the dream begins}

The sky is vast, the exploration is endless, and the gas giant planet Saturn is explored. From the 1970s, the "Pioneer-11, Voyager-2, Cassini-Huygens" probes were launched toward Saturn and flew over Jupiter, U ranus, Neptune. In 2004, the "Huygens-Landing Module" escaped from the "Cassini-orbiting spacecraft" and reached 1,270 kilometers above the sixth satellite of Saturn, crossing the atmosphere and successfully landing softly.

\subsection{New space probes to expand the future space for human beings}

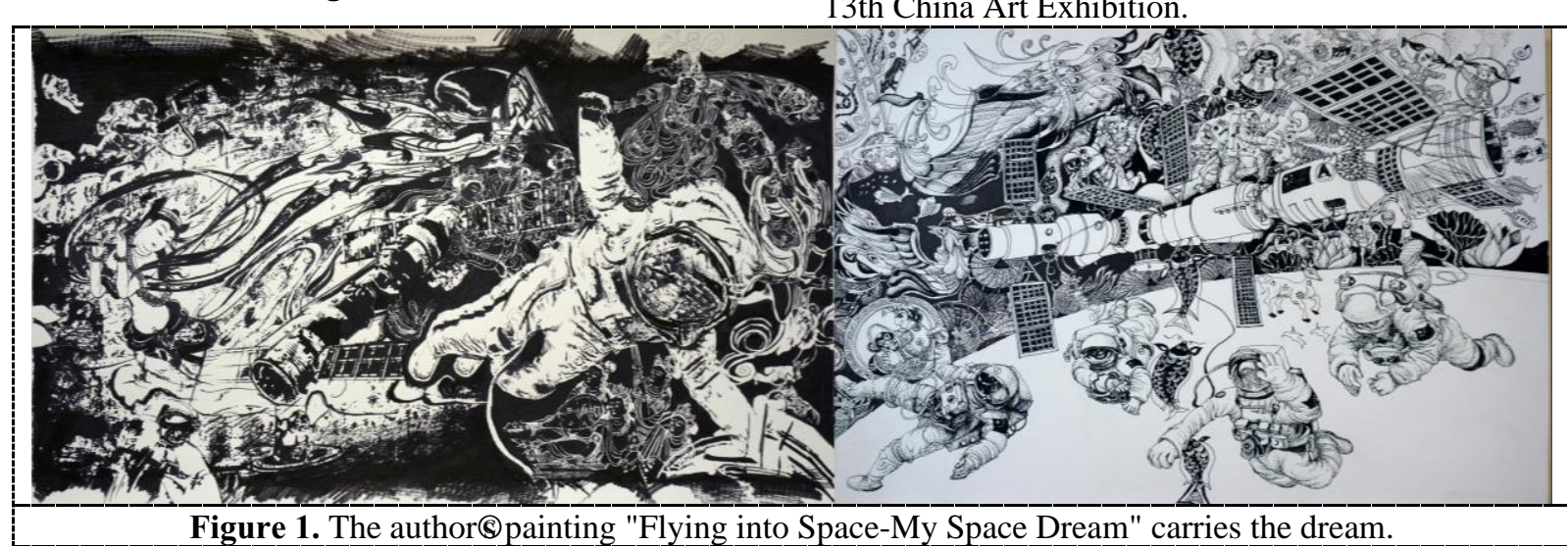

Corresponding author's e-mail: 493102441@ qq.com

phone: 13838838132
A great cause begins with dreams, and dreams are a source of vitality. From 2020 to 2030, mankind will implement 16 space missions of solar system planets (satellites), including China-M ars exploration, Tiangong space station, Europe-landing moon, Japan-asteroid sampling and return. The past honest detectors have the same appearance, clumsy and mediocre shape, and have nothing to do with the aesthetic hall [1]. The new type of detector uses aesthetics to express the mechanical structure, integrates culture, and displays the smart industrial design beauty. Exploring the vast universe is a relentless pursuit of humankind, and aerospace must reflect the colorful local cultural characteristics of different countries. The author combines ancient art (paper-cuts, shadow puppets, murals) and aerospace. "A erospace Dream" won the 2018 "China@ First Illustration Painting Exhibition" (Figure 1), and was selected as the sixth China Y outh Art Exhibition, The 13th China A rt Exhibition. 


\section{The new detector consists of a relay satellite, a landing capsule, and a cruise vehicle (Saturn car)}

\subsection{Titan's geological environment looks like early Earth}

Saturn $\odot$ largest satellite has a thick atmosphere, with land, lakes, mountains and plants, much like the Earth 100 million years ago, and it has the same environment as Jupiter@ second satellite (the world@ oceans under the thick ice). A re satellites with unique geological structures, which are of great significance for studying the solar system.

\subsection{Achieving the three goals of "surround, landing, and cruising"}

French designer Dassault once said: Only beautiful aircraft can be good aircrafts [2]. "Titan N ew Detector" is a huge mechanical body composed of many parts, including relay satellites, landing capsules and Saturn vehicles (Figure 2). It is 10 meters long and 5 meters wide and weighs 10.81 tons after fueling. It used an ion thruster to fly, and was captured by Saturn $\Theta$ gravity after six years. The relay satellite separated from the landing capsule and surrounded Titan to serve as a communication relay station. The landing capsule entered the atmosphere, landed softly on the ground, and released the Saturn rover to begin global observations. Due to the atmosphere, the landing module can be decelerated with three parachutes, while the variable thrust assist engine
$(9190 \mathrm{~N})$ is used to adjust the power to complete the "correction, braking, and descent" control. At 108 meters above the ground, hover in the air, the radar scans the surface, pans, avoids large pits and obstacles, looks for new landing points, and does not land until the safe area. The Saturn weighs 3.22 tons. It follows the mechanical slide and slowly moves from the landing platform to the ground and begins cruising.

\subsection{Quantum communication embodies the ultimate manual mechanical aesthetics}

There are 7 types of landing capsules and 9 scientific instruments on Saturn, which can accurately collect data. Seismometer - measure core thickness, mantle density and crustal structure, fluid dynamics instrument-drill 3.8 meters below the soil, measure internal temperature, judge core is solid (liquid), three-dimensional structure and dynamic measuring instrument-measure magnetic field, carbon A nalytical laboratory - detection of surface mineral composition and geological history, monitoring of spontaneous decay of water atom protons, AA atmospheric analyzer - study of the atmosphere (clouds, haze), biological experiment chamber-detection of signs of methane microorganisms, Higgs boson-dion detector- Detecting the space distortion caused by Saturn $\odot$ huge gravitational force, and judging the existence of long fiber structure dark matter (dark matter hair), Hyper-mini detector) —observing the "neutrino beam" of the sun and supernova, QC quantum communication - testing entangled transmission at long distances .

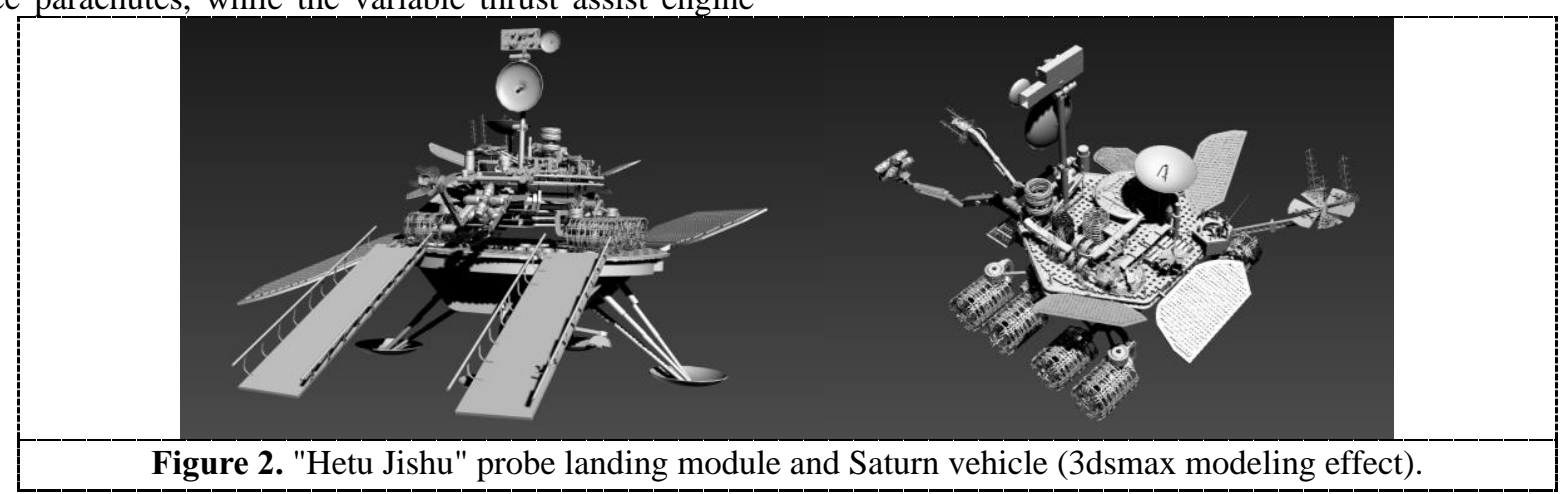

Figure 2. "Hetu J ishu" probe landing module and Saturn vehicle (3dsmax modeling effect).

\section{Saturn vehicle is a combination of artistic appearance and technical rationality}

\subsection{Design of "vibration reduction platform" mimicking biology, rigid body dynamic aesthetics}

The central support platform draws on the crawling posture of the natural creature "turtle" and designs three "vibration-absorbing matrix platforms" based on the principle of biological imitation. The air cylinder controls the spring to move up and down, compensates the vibration frequency, avoids obstacles flexibly, and effectively crosses bumpy stones and puddles. Made of memory metal, capable of bending and torque, intelligent skinning in a short time, self-healing, maintaining geometry, maintaining high strength support, resisting fatigue, and maintaining traction when passing through soft sand and hard rocks.

\subsection{Power traction design (drive) embodies the process aesthetics of manufacturing technology}

The surface temperature of Titan is minus 179.2 degrees Celsius, methane liquefies to form clouds, rises to high 
altitude and turns into rain and snow, and then falls to the ground, filling the surface depressions and craters, making the surface of the planet smooth. M ethane rain forms a lake with a maximum area of 400,000 square kilometers, and waves and tides are very weak, less than a dozen centimeters. Saturn requires the ground contact area of eight wheels to increase friction to prevent skids and runaways. The wheels wear while driving, and are corroded and burned by the surface methane. The connecting rods and gears show a mottled gloss.

\subsection{Honeycomb mesh section wheel, seamless casting using 3D printing technology}

Wheels are cast using 3D printing (metal printing, vacuum cold welding). The wheels have a diameter of $58 \mathrm{~cm}$, a width of $81 \mathrm{~cm}$, a weight of 1919 grams, an independent motor drive, can climb 45-degree slopes, and a speed of 81 meters per hour. It does not use a traditional blade-like design, but uses a honeycomb stacked mesh surface (claw layer) design (Figure 3), because the soil on the planet( surface is very different from the inner particles, and large stones are deposited inward by gravity, and the ground is covered by A fter the car is pressed, an indentation will be formed, and the powder soil will fall into the gap, exposing a thicker bulky gravel-pointed stone, which will jam the blade-like wheels, and the honeycomb wheels have good elasticity, discharge sludge, and will not slip and sink.

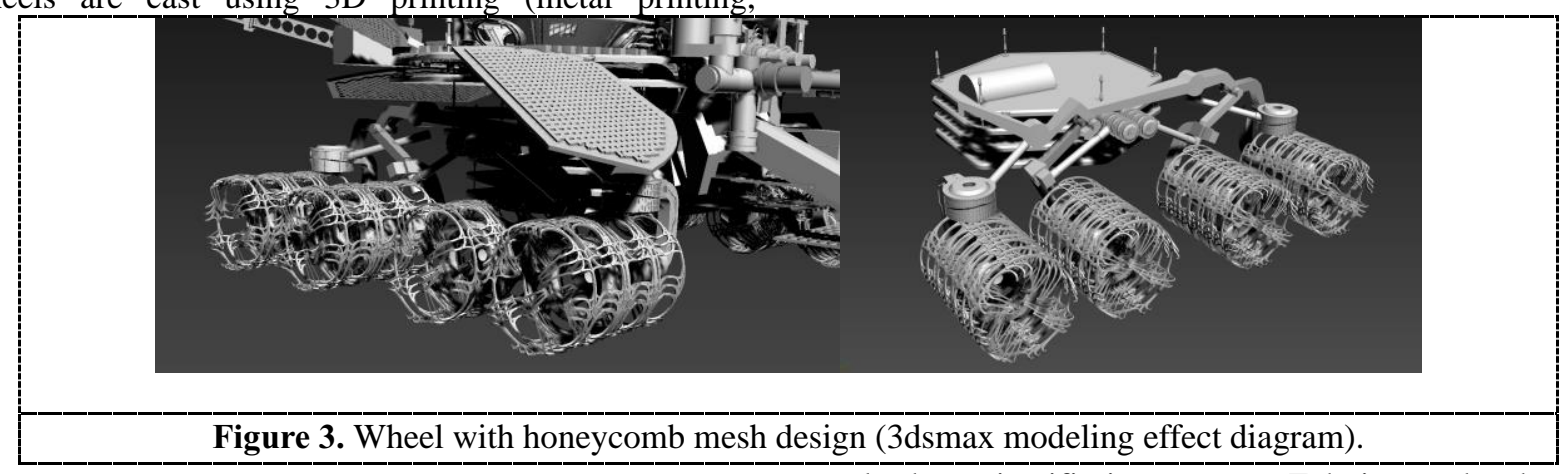

\subsection{Design of solar, isotope thermal energy generator and graphene hybrid battery}

Too far from the sun, the solar power generation efficiency is low, and sand storms and rain and snow will also cover up the solar panels. Therefore, it is also equipped with isotope heat generators and graphene batteries. The generator is a plutonium-238 circular device, separated by a green barrier to prevent radiation, zero radiation, zero pollution, using the "West Baker effect" to release neutrons and heat, which converts heat into electricity and provides 618 watts of power. At minus 179.2 degrees Celsius, the battery can provide enough heat to ensure that the scientific instrument "wraps in sleep", is not frozen and can wake up on its own.

\section{Aerospace design is no longer a simple imagination, but a smart, stylish art symbol}

\subsection{Manual installation of "quantum communication", the ultimate in mechanical aesthetics}

In addition to the traditional wireless microwave, the detector also has quantum communication. The "quantum entanglement-secret key" communication device consists of 3081 parts, 24 pairs of gears, and realizes laser "invisible transmission" in an $8.1 \mathrm{~cm}$ radius interferometer. It is a spiral mechanical structure surrounding a cylinder and other scientific instruments. Echoing each other, the shape is beautiful and integrated. "Quantum invisible teleportation, high-speed interference of satellite-ground laser communication" is an open umbrella shape, simple and elegant. Engineers manually installed 919 optical components to the "long-distance quantum entanglement distribution antenna". The huge workload and micron precision are the ultimate embodiment of mechanical aesthetics.

\subsection{Lonely Explorer, Aesthetic Design of 3D Four-Dimensional Robotic Arm}

Saturn ( exquisite car manufacturing technology is reflected in the design of the pivot and mast of the "3D four-dimensional robotic arm" (Figure 4). It consists of tw o brackets with a length of 1.6 meters. On the neck and head of the five-mast mast, navigation cameras and chemical spectrometers are installed (to induce the laser to irradiate and evaporate a portion of the rocks and soil to obtain samples). Terminal instruments also include: drilling machines, rock brushes, holographic cameras, al pha particle $X$-ray spectrometers.

\subsection{Titan's intelligent brain- neural chain Ai chip and autonomous driving computer}

The "relay communication satellite" staying in orbit transfers surface data for the traveling "Saturn vehicle" for sharing and collaboration. Because driving in a harsh environment for a long time, the hardware is easy to age, and a backup computer is needed [3]. It uses a high-density neural chain stereo chip, eliminating a large 
number of cables, thereby reducing the volume occupied by the control machinery. Its brain is a computer composed of a neural chain Ai chip and a big data sharing network, a 3D holographic camera and a structured wall panel conductor all over the body. It uses fiber optics to densely connect all terminal equipment, all around the landing capsule and Saturn (Figure 5) With primary self-awareness and logical thinking, using rare metals such as "rhenium, iridium, Ianthanum, osmium" and "silicon-graphene-germanium" composite materials for autonomous driving.
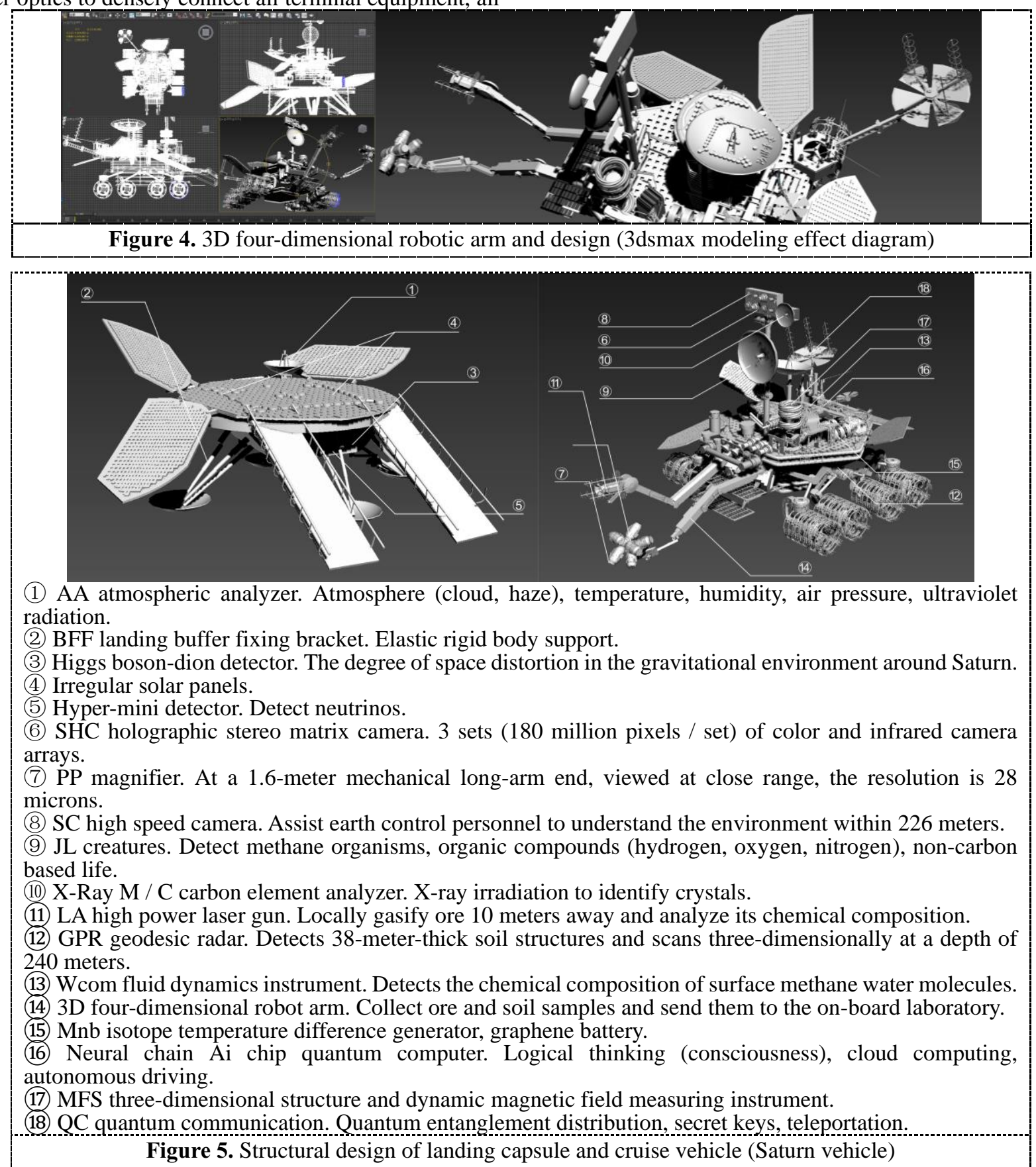

\section{Enlightenment of future spaceflight}

The Titan detector does not have an icy mechanical structure, but fully absorbs the cultural symbols of the "Dunhuang Flying Sky" in the east (Figure 6), and uses the ancient wisdom philosophy of "Hetu Jishu-Zhou Y i" (Figure 7) to carry out preliminary ideas. The final design of the aerospace product integrating aesthetics, technology and $\mathrm{Ai}$ intelligence was designed, showing beautiful lines and exquisite mechanical aesthetics. It is a whole, echoing each other, elegant and concise, exudes dynamic vitality. It is predicted that in the future, it will be invisible (holographic laser stereoscopic display), it will have a magnetic sail engine, the spacecraft will surf in the magnetic field around the planet, change the orbit through the interaction of the magnetic field, use nuclear impulse acceleration, and the laser will propel the light sail. Bending time and space to achieve the speed of light, a single silicon wafer is used to produce highly integrated tiny satellites, which form a mirror with a diameter of several kilometers to illuminate the back of the earth. To explore the universe, only by cooperating with 
international space and focusing on the wisdom of all mankind, can we create a better future.
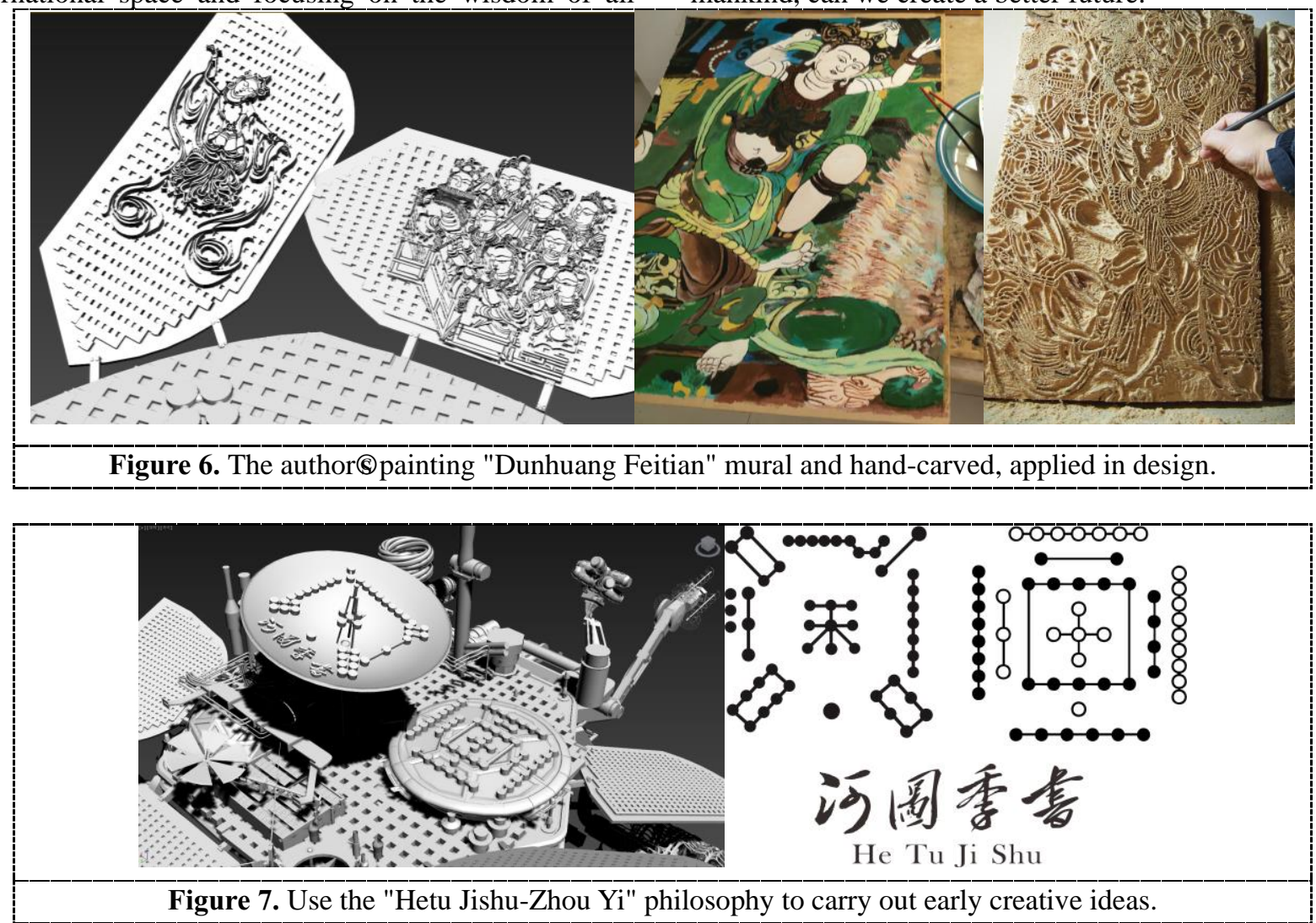

\section{References}

[1] Songfeng Zhang. (2001) The future of aircraft industry design. Decoration, 80-81

[2] Jing Gao. (1999) Explore the development direction of aerospace machinery design and manufacturing and automation. China A erospace, 21-22

[3] Qi Wang. (1997) Scanning of Aircraft Industrial Design Industry. Journal of Construction Machinery, 100-106 